\title{
Correction to: The characteristic cycle and the singular support of a constructible sheaf
}

\section{Takeshi Saito ${ }^{1}$}

Published online: 3 April 2019

(C) Springer-Verlag GmbH Germany, part of Springer Nature 2019

Correction to: Invent. math. (2017) 207:597-695 https://doi.org/10.1007/s00222-016-0675-3

The first part of Proposition 7.4 and its proof in pp. 670-671 should be corrected as follows. The author apologizes for the mistake.

Proposition 7.4 (Beilinson) Let $\mathbf{P}=\mathbf{P}^{n}$ be a projective space, and let $\mathbf{P}^{\vee}$ be the dual projective space. Let $\mathcal{G}$ be a constructible complex of $\Lambda$-modules on $\mathbf{P}^{\vee}$, and let $\mathcal{F}$ denote the naive inverse Radon transform $R \boldsymbol{p}_{*} \boldsymbol{p}^{\vee *} \mathcal{G}$. Let $C^{\vee} \subset T^{*} \mathbf{P}^{\vee}$ be a closed conical subset such that every irreducible component is of dimension $n$, and let $C=\boldsymbol{p}_{\circ} \boldsymbol{p}^{\vee} C^{\vee} \subset T^{*} \mathbf{P}$. Assume that $\mathcal{G}$ is microsupported on $C^{\vee} \subset T^{*} \mathbf{P}^{\vee}$.

Let $X$ be a smooth subscheme of $\mathbf{P}$, and assume that the immersion $h: X \rightarrow$ $\mathbf{P}$ is properly $C$-transversal. Using the notation in (3.10), let $p: X \times \mathbf{P} Q \rightarrow X$ be the projection and $p^{\vee}: X \times_{\mathbf{P}} Q \rightarrow \mathbf{P}^{\vee}$ be the restriction of $\boldsymbol{p}^{\vee}$.

1. We have

$$
\mathbf{P}\left(C C R p_{*} p^{\vee *} \mathcal{G}\right)=\mathbf{P}\left(p_{!} C C p^{\vee *} \mathcal{G}\right)=\mathbf{P}\left(p_{!} p^{\vee !} C C \mathcal{G}\right)
$$

The original article can be found online at https://doi.org/10.1007/s00222-016-0675-3.

$\triangle$ Takeshi Saito

t-saito@ms.u-tokyo.ac.jp

1 Department of Mathematical Sciences, University of Tokyo, Tokyo 153-8914, Japan 
In particular, for $X=\mathbf{P}$, we have

$$
\mathbf{P}(C C \mathcal{F})=\mathbf{P}\left(\boldsymbol{p}_{!} C C \boldsymbol{p}^{\vee *} \mathcal{G}\right)=\mathbf{P}\left(\boldsymbol{p}_{!} \boldsymbol{p}^{\vee !} C C \mathcal{G}\right) .
$$

2. We have

$$
C C h^{*} \mathcal{F}=h^{!} C C \mathcal{F} .
$$

Proof 1. First, we prove the second equality in (7.4) for properly $C$-transversal immersion $h: X \rightarrow \mathbf{P}$. By Corollary 3.13.2, $p^{\vee}: X \times \mathbf{P} Q \rightarrow \mathbf{P}^{\vee}$ is $C^{\vee}$ transversal and hence $p^{*} \mathcal{G}$ is micro-supported on $p^{\vee} C^{\vee}$. Since $p^{\vee}: X \times \mathbf{P}$ $Q \rightarrow \mathbf{P}^{\vee}$ is smooth outside $\Delta_{X} \subset X \times_{\mathbf{P}} Q$ (3.11), we have $C C p^{\vee *} \mathcal{G}=$ $p^{\vee} C C \mathcal{G}$ outside $\Delta_{X} \subset X \times_{\mathbf{P}} Q$ by Proposition 5.17. By the assumption that $h: X \rightarrow \mathbf{P}$ is $C$-transversal, the pair $\left(p^{\vee}, p\right)$ is $C^{\vee}$-transversal on a neighborhood of $\Delta_{X} \subset X \times_{\mathbf{P}} Q$ by Corollary 3.13.1 (1) $\Rightarrow(2)$. Hence, we have the second equality in (7.4).

We prove the first equality in (7.4). We may assume that $k \ldots$

(We keep from the 3rd line of p. 671 to the displayed formula as it is.)

$$
\phi_{u}\left(R p_{*} p^{\vee *} \mathcal{G}, f\right) \rightarrow R \Gamma\left(Q \times_{X} u, \phi\left(p^{\vee *} \mathcal{G}, f p\right)\right) \rightarrow \bigoplus_{v} \phi_{v}\left(p^{\vee *} \mathcal{G}, f p\right) .
$$

For equalities (7.2), it suffices to take $X=\mathbf{P}$.

(From the beginning of the proof of 2. on, no change is necessary.)

Publisher's Note Springer Nature remains neutral with regard to jurisdictional claims in published maps and institutional affiliations. 